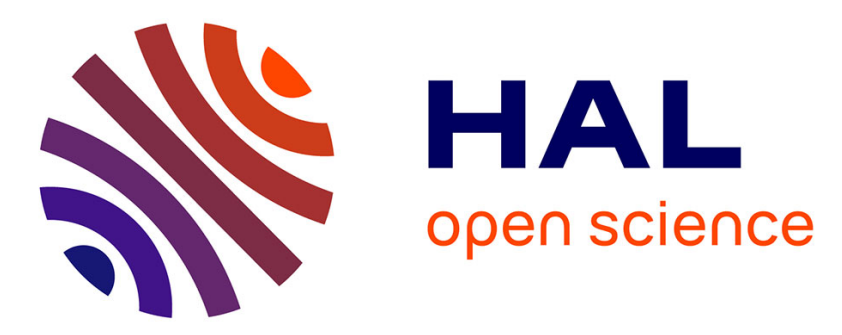

\title{
An Integral Formulation for the Computation of 3D Eddy Current Using Facet Elements
}

Thanh Trung Nguyen, Gérard Meunier, Jean-Michel Guichon, Olivier Chadebec, T.S. Nguyen

\section{- To cite this version:}

Thanh Trung Nguyen, Gérard Meunier, Jean-Michel Guichon, Olivier Chadebec, T.S. Nguyen. An Integral Formulation for the Computation of 3D Eddy Current Using Facet Elements. IEEE Transactions on Magnetics, 2014, 50 (2), 10.1109/TMAG.2013.2282957 . hal-00957440

\section{HAL Id: hal-00957440 https://hal.science/hal-00957440}

Submitted on 2 Dec 2020

HAL is a multi-disciplinary open access archive for the deposit and dissemination of scientific research documents, whether they are published or not. The documents may come from teaching and research institutions in France or abroad, or from public or private research centers.
L'archive ouverte pluridisciplinaire HAL, est destinée au dépôt et à la diffusion de documents scientifiques de niveau recherche, publiés ou non, émanant des établissements d'enseignement et de recherche français ou étrangers, des laboratoires publics ou privés. 


\title{
An Integral Formulation for the Computation of 3D Eddy Current Using Facet Elements
}

\author{
T-T. Nguyen ${ }^{1}$, G. Meunier ${ }^{1}$, J-M. Guichon ${ }^{1}$, O. Chadebec ${ }^{1,2}$ and T-S. Nguyen ${ }^{1}$ \\ ${ }^{1}$ Grenoble Electrical Engineering Laboratory, University of Grenoble \\ Grenoble-INP / Université Joseph Fourier / CNRS UMR 5269, Grenoble, France \\ ${ }^{2}$ GRUCAD/EEL/CTC/UFSC, Universidade Federal de Santa Cantarina, Brazil
}

\begin{abstract}
A volume integral formulation to compute eddy currents in non-magnetic conductive media is presented. The current distribution is approximated with facet finite elements. The formulation is general and leads to an equivalent lumped elements circuit. In order to ensure the solenoidality of the current distribution, an algorithm detecting the independent loops is then used for the resolution. The formulation is tested on TEAM workshop Problem 7. Even with coarse meshes, its accuracy is demonstrated.
\end{abstract}

Index Terms - Eddy currents, Facet element, Generalized PEEC method, Integral equation method.

\section{INTRODUCTION}

$\mathrm{T}_{\mathrm{i}, \mathrm{s}}$ HE PEEC method (Partial Element Equivalent Circuit) is a well-known integral equation technique leading to an equivalent circuit representation of a electromagnetic device. It is mainly used for the modeling of complex interconnect problems and can be favorably applied to devices where the air region is dominant [1]. However, the classical PEEC method does not enable easily the modeling of 3D conductive media. Moreover, it requires the generation of a structured mesh associated with uniform current density on each element. Thus, it use is still limited for general geometries.

Previously, eddy currents integral formulations based on general Whitney elements have been proposed [12][13]. Our approach has the particularity of being based on the use of facet elements [2]. The reliability of such elements has already been shown for finite element magnetostatic formulations [3], for integral magnetostatic formulation [4] and also for steady conduction problems [5]. Different quantities have been approximated, such as the magnetization in [4] and the current distribution in [3][5].

This paper proposes a generalization of the classical inductive PEEC formulation using facet elements enabling the use of general mesh. A similar approach has been recently proposed by Alotto et al. but limited to the modeling of surface regions [6]. In our approach, the current density is linearly interpolated with first order facet elements. An equivalent electrical circuit representation, whose branches are the facets and the nodes of these branches are the centroids of elements, is proposed (i.e. an equivalent electrical circuit build on the dual mesh). The integral inductive PEEC formulation is then adapted to this configuration. The solenoidality of the current distribution is ensured thanks an independent loops search technique.

\section{FORMULATION}

\section{A. PEEC Integral equation}

From Maxwell-Faradays equation, a magnetic vector $\mathbf{A}$ and a scalar electric potential U exist such as:

$$
\mathbf{E}=-j \omega \mathbf{A}-\operatorname{grad} U
$$

where $\mathbf{E}$ is the electric field and $\omega$ the pulsation.

We consider the conductive and non-magnetic region $\Omega_{C}$ representing a set on conductors. The electric field $\mathbf{E}$ in the conductor satisfies Ohm's law

$$
\frac{\mathbf{J}}{\sigma}=-j \omega \mathbf{A}-\operatorname{grad} U
$$

with $\sigma$ is the material conductivity and $\mathbf{J}$ is the current density. Without any magnetic material, we can write the magnetic vector potential $\mathbf{A}$ as

$$
\mathbf{A}=\frac{\mu_{0}}{4 \pi} \int_{\Omega_{C}} \frac{\mathbf{J}}{r} d \Omega_{C}
$$

where $\mu_{0}$ is the vacuum permeability. By introducing this expression in (2), we get

$$
\frac{\mathbf{J}}{\sigma}+\frac{j \omega \mu_{0}}{4 \pi} \int_{\Omega_{C}} \frac{\mathbf{J}}{r} d \Omega_{C}=-\operatorname{grad} U
$$

The current density has to satisfy the several conditions. In the conductive region $\Omega_{\mathrm{C}}$,

$$
\operatorname{div} \mathbf{J}=0
$$

and on the boundary of $\Omega_{C}$,

$$
\mathbf{n . J}=0
$$

where $\mathbf{n}$ is the external normal.

\section{B. Facet Element Interpolation}

The current density is interpolates with first order facet elements such as

$$
\mathbf{J}=\sum_{j} \mathbf{w}_{j} \mathbf{I}_{j}
$$


where $\mathbf{w}_{j}$ is shape function and $\mathrm{I}_{j}$ is flux across the $j$ th facet. Expressions for $\mathbf{w}_{j}$ can be easily computed for different kind of element [2]. The main property of such element family is that the normal component of $\mathbf{w}_{j}$ is conserved through each facet, so also ensuring the conservation of current.

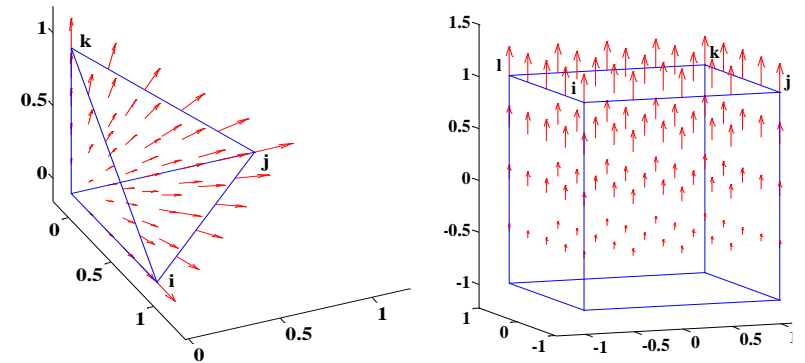

Fig. 1. Representation of shape functions of the faces $\{i, j, k\}$ and $\{i, j, k, 1\}$ for the reference tetrahedral and reference hexahedra.

Let us remember some the properties for these shape functions:

$$
\begin{aligned}
& \mathbf{w}_{j} \cdot \mathbf{n}= \pm \frac{1}{\mathrm{~s}_{j}} \\
& \operatorname{div}_{j}= \pm \frac{1}{\mathbf{v}_{e}}
\end{aligned}
$$

where $\mathrm{s}_{j}$ is surface of facet $j$ and $\mathrm{v}_{e}$ is volume of element $e$ which contains the facet $j$. The sign $( \pm)$ depends on the facet orientation.

\section{System Assembly Using Facet Elements}

Applying the Galerkin method to (4) by using $\mathbf{w}_{i}$ as projection functions, a system of linear equations is obtained

$$
\left[\mathbf{Z}_{\mathbf{b}}\right] \mathbf{I}_{\mathbf{f}}=([\mathbf{R}]+j \omega[\mathbf{L}]) \mathbf{I}_{\mathbf{f}}=\mathbf{U}_{\mathbf{b}}
$$

with

$$
\begin{gathered}
\mathrm{R}_{i j}=\int_{\Omega_{C}} \frac{\mathbf{w}_{i} \cdot \mathbf{w}_{j}}{\sigma} d \Omega_{C} \\
\mathrm{~L}_{i j}=\frac{\mu_{0}}{4 \pi} \int_{\Omega_{C}} \mathbf{w}_{i} \cdot \int_{\Omega_{C}} \frac{\mathbf{w}_{j}}{r} d \Omega_{C} d \Omega_{C} \\
\mathrm{U}_{b i}=-\int_{\Omega_{C}} \mathbf{w}_{i} \cdot \operatorname{grad} U d \Omega_{C}
\end{gathered}
$$

Matrix $\left[\mathbf{Z}_{\mathbf{b}}\right]$ can be seen as the impedance matrix of the electrical equivalent circuit generated, $\mathbf{I}_{\mathbf{f}}$ is vector of currents through the facets, $[\mathbf{R}]$ is matrix of the resistive terms, and [L] is the matrix of the mutual inductances. Let us notice that the resistive matrix $[\mathbf{R}]$ is sparse and similar to a matrix obtained during a classical FEM assembly. On the other hand, the inductive part $[\mathbf{L}]$ is fully dense and more representative of integral method-based assembly. To avoid integral singularity inaccuracies while the computation of $[\mathbf{L}]$, different Gauss points repartition are considered especially for the computation of the self inductance (i.e. $\mathrm{L}_{i i}$ ).
Let us define $\mathrm{U}_{e}$ the average values of the voltage on each finite volume element $\Omega_{e}$ and $\mathrm{U}_{f}$ its average value on each face $\Gamma_{\mathrm{f}}$ of the mesh. We have:

$$
\begin{aligned}
\mathrm{U}_{e} & =\frac{1}{\mathrm{v}_{e}} \int_{\Omega_{e}} U d \Omega_{e} \\
\mathrm{U}_{f} & =\frac{1}{\mathrm{~s}_{f}} \int_{\Gamma_{f}} U d \Gamma_{f}
\end{aligned}
$$

with $\mathrm{v}_{e}$ is the volume of element $e$ and $\mathrm{s}_{f}$ is surface of facet $f$. Let us apply divergence theorem on equation (13), we get

$$
\mathrm{U}_{b i}=-\int_{\Gamma}\left(\mathbf{w}_{i} \cdot \mathbf{n}\right) U d \Gamma+\int_{\Omega_{C}} \operatorname{div}\left(\mathbf{w}_{i}\right) U d \Omega_{C}
$$

where $\Gamma$ is the boundary of region $\Omega_{C}$.

Let us now consider the computation of $\mathrm{U}_{b i}$ for an internal facet $i$ (see Fig. 2.). The first term in (16) is null because the value of $\mathbf{w}_{\mathbf{i}}$ vanishes on all others facets of both adjacent elements sharing facet $i$. Thus, from (9) and (14), we can deduce that $\mathrm{U}_{b i}$ is the difference between the averaged voltages of both elements sharing $i$.

Let us now consider $\mathrm{U}_{b i}$ for a border facet (i.e. belonging to the boundary of region $\Omega_{C}$ ). From (8) and (15) the value of the first term in (16) becomes equal to the averaged potential on the facet. The second term becomes equal to the volume averaged voltage on the only elements to whom facet $i$ belongs. Of course, facet orientations have to be considered in order to properly compute the values of $\mathrm{U}_{b i}$.

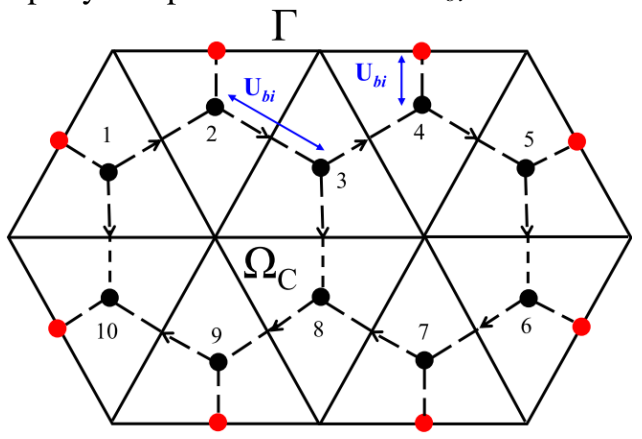

Fig. 2. Primal and dual meshes, the black points are the centroid of elements, red ones are the centroid of the faces on the boundary. Both $\mathrm{U}_{b i}$ configurations are represented (internal and border facets).

From previous considerations, an equivalent electrical circuit (10) can be generated. The branches of this circuit are represented by the facets of initial mesh. Each element of the mesh can then be seen as a node of this circuit. This electrical circuit is in fact an equivalent representation of the dual mesh.

\section{Resolution of the Electrical Circuit}

In order to ensure the solenoidality of $\mathbf{J}$, we can use a similar technique to which used for classical structured PEEC method. In our approach, we have decided to use a circuit solver resolution based on the determination of independent loops. The general principle consist in find small topological loops (minimum number of branches), allowing a very quick research of independent loops. Furthermore, the choice to 
maximize the number of small loops allows significantly improving the matrix condition number of the final system of equations.

In addition, we can notice that the boundary condition (5) can be easily taken into account in the formulation, by simply suppressing the associated degree of freedom in the equivalent circuit. If the meshed conductor is electrically coupled with an external circuit, the electric coupling can naturally be imposed in the formulation without any difficulty.

Fundamental circuit equations are expressed in the presence of external source voltages.

$$
[\mathbf{M}]\left(\mathbf{U}_{\mathbf{b}}+\mathbf{U}_{\mathbf{s}}\right)=0
$$

with $[\mathbf{M}]$ is the branch-fundamental independent loop transition matrix where the value of each element can be $-1,0$ or 1 and $\mathbf{U}_{\mathrm{s}}$ the vector of external voltage sources (most part of time equal to 0 ). We can write a new system of linear equations where unknowns $\mathbf{I}_{\mathbf{m}}$ are currents flowing in independent loops.

$$
[\mathbf{M}]\left[\mathbf{Z}_{\mathbf{b}}\right][\mathbf{M}]^{\mathbf{t}} \mathbf{I}_{\mathbf{m}}=-[\mathbf{M}] \mathbf{U}_{\mathbf{S}}
$$

Once linear system has been solved, we obtain the currents flowing on each branch by applying the following equation.

$$
\mathbf{I}_{\mathbf{m}}=[\mathbf{M}]^{\mathrm{t}} \mathbf{I}_{m}
$$

\section{E. Addition external source field}

In presence of an external source like (a coil for instance) in which the current density is imposed, equation (4) has to be adapted.

$$
\frac{\mathbf{J}}{\sigma}+\frac{j \omega \mu_{0}}{4 \pi} \int_{\Omega_{C}} \frac{\mathbf{J}}{r} d \Omega_{C}+j \omega \mathbf{A}_{0}=-\operatorname{grad} U
$$

where $\mathbf{A}_{\mathbf{0}}$ is the source magnetic vector potential created by the external source. Equation (10) becomes

$$
\left[\mathbf{Z}_{\mathbf{b}}\right] \mathbf{I}_{\mathbf{f}}=\mathbf{U}_{\mathbf{b}}+\mathbf{U}_{\mathbf{0}}
$$

where

$$
\mathrm{U}_{0 i}=-j \omega \int_{\Omega_{C}} \mathbf{w}_{i} \cdot \mathbf{A}_{0} d \Omega_{C}
$$

Independent loops search technique and resolution is then applied to (20) as previously.

\section{NUMERICAL EXAMPLE}

In order to validate the proposed formulation and to show its performances, we consider the Problem 7 of the TEAM workshop benchmark problems [8]. The results will be compared to those obtained with FLUX [9], a commercial Finite Element Method (FEM) program.

The geometry of the Problem 7 is presented in Fig. 3. The problem consists in an asymmetrical conductor with a hole and an exciting coil. The coil is placed $30 \mathrm{~mm}$ above the plate and excited with 2472 Ampere-turns at $50 \mathrm{~Hz}$ and $200 \mathrm{~Hz}$. The driving current reaches the maximum at a phase of 0 degrees. In this problem, we have to add an additional source magnetic vector potential representing the contribution of the coils in which the current distribution is known. We have

$$
\mathbf{A}_{0}=\frac{\mu_{0}}{4 \pi} \int_{\Omega_{0}} \frac{\mathbf{J}_{0}}{\mathrm{r}} \mathrm{d} \Omega_{0}
$$

where, $\mathbf{J}_{0}$ is the current density vector of the coil and $\Omega_{0}$ is coil region. $\mathbf{A}_{\mathbf{0}}$ is evaluated numerically thank a tetrahedral discretization of the coil and a Gauss point quadrature.

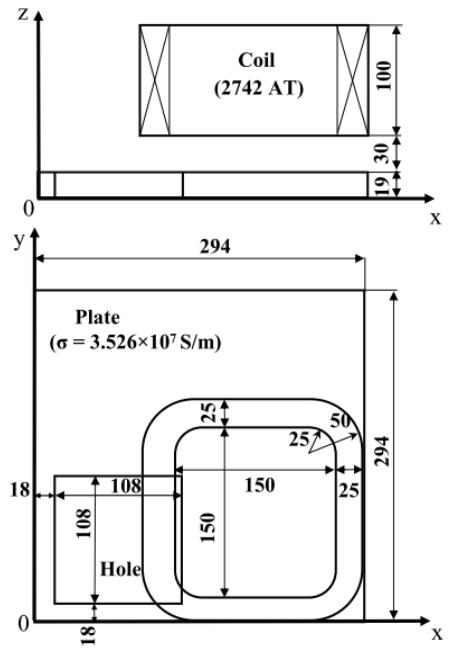

Fig. 3. Geometry of TEAM problem 7: coils and conducting plate with hole (all dimension are in $\mathrm{mm}$ ).

We focus on the computed eddy current distribution and losses in the plate (Fig. 4(a), Fig. 4(b) and Tab. 1.). The results show that the relative error of the formulation in comparison with the 3D FEM associated to a very high mesh density is good $(<5 \%)$. Figure 5(a) and 5(b) present the convergence of the Joules losses value of two methods follows the number of elements in the plate at $50 \mathrm{~Hz}$ and $200 \mathrm{~Hz}$. Results provided by our formulation are very encouraging, the convergence being reached with a very few number of elements in comparison with the FEM one.

Another advantage of the new formulation is its capacity to treat any multiply connected problems thanks to the efficient determination of independent loops of the equivalent electrical circuit. This point is really valuable for the modeling of industrial problem, where the techniques of automatic cuts search can be poorly efficient [10].

TABLE I

RELATIVE ERROR OF OUR FORMULATIONS COMPARED TO THE FEM SOLUTION

\begin{tabular}{|l|c|c|c|c|}
\hline & \multicolumn{2}{|c|}{$\mathrm{f}=50 \mathrm{~Hz}$} & \multicolumn{2}{c|}{$\mathrm{f}=200 \mathrm{~Hz}$} \\
\cline { 2 - 5 } & $\begin{array}{c}\text { Joules } \\
\text { Loss(W) }\end{array}$ & Diff. & $\begin{array}{c}\text { Joules } \\
\text { Loss(W) }\end{array}$ & Diff. \\
\hline $\begin{array}{l}\text { FEM 3D with } \\
2.000 .000 \text { elements }\end{array}$ & 4.70 & Ref. & 9.50 & Ref. \\
\hline $\begin{array}{l}\text { Integral formulation } \\
\text { with 7000 elements }\end{array}$ & 4.78 & $1.7 \%$ & 9.70 & $2.1 \%$ \\
\hline
\end{tabular}




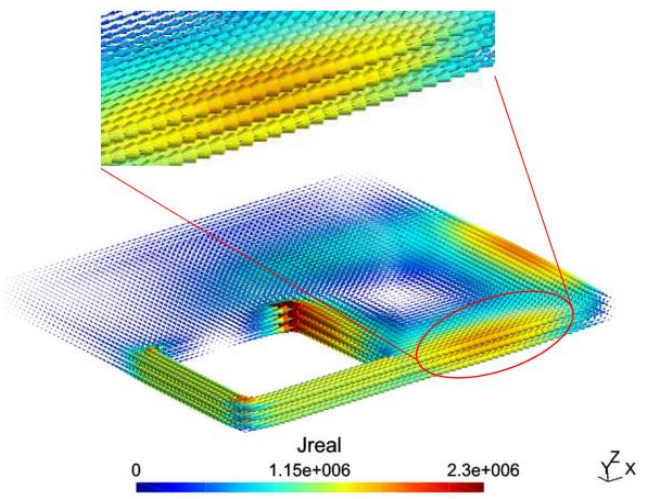

(a)

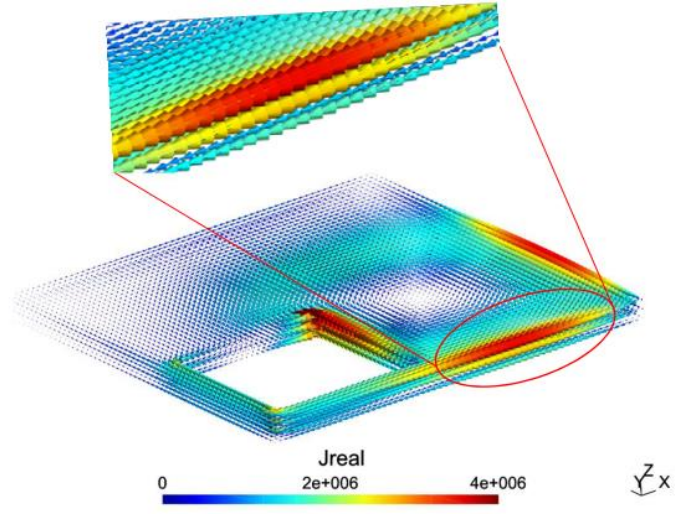

(b)

Fig. 4. Eddy current distribution in the plate, (a) at $50 \mathrm{~Hz}$, (b) at $200 \mathrm{~Hz}$

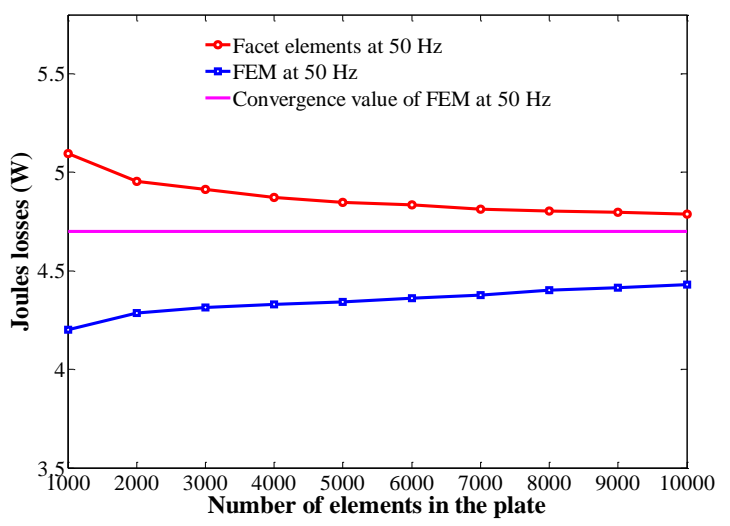

(a)

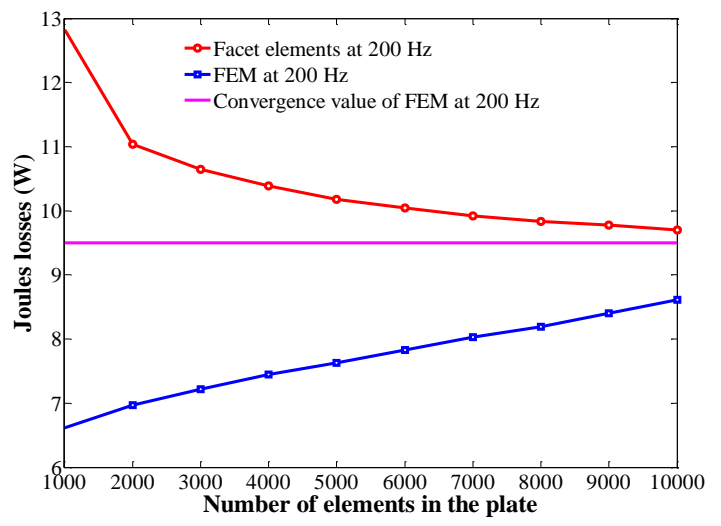

(b)

Fig. 5. Convergence of Joules losses value in the plate, (a) at $50 \mathrm{~Hz}$, (b) at $200 \mathrm{~Hz}$

\section{CONCLUSION}

In this paper, we have presented an original eddy currents volume integral formulation using facet elements dedicated to the modeling of non magnetic conductors. The formulation has a small relative error in comparison with a $3 \mathrm{D}$ reference FEM with a high mesh density. Moreover, the convergence of the results is quickly reached and results with very coarse meshes remain acceptable.

This new formulation seems very attractive because it enables an easy treatment of the multiply connected problems without cuts technique and without meshing the air. Moreover, the coupling with the classical PEEC formulation is natural, both formulation having same theoretical bases. It opens interesting perspective for the modeling for multiply connected volume/surface regions with direct electrical connections a complex wiring system.

The obtained matrix is fully dense but this problem can be overcome by the use of a compression technique like Fast Multipole Method [11] enabling the save of memory and reducing the computation time. It remains to study the robustness of this approach in this context where convergences can sometimes be difficult to reach.

\section{REFERENCES}

[1] A. E. Ruehli, "Equivalent circuit models for three dimensional multiconductor systems," IEEE Trans. Microwave Theory and Tech., vol. 22, no.3, pp. 216-221, Mar. 1974.

[2] A. Bossavit, "Whitney forms: a class of finite elements for three dimensional computations in electromagnetism," IEE Processing Pt.A, vol.135, no.8, pp.493-500, Nov. 1998.

[3] Y. Le Menach, S. Clenet, and F. Piriou, "Determination and utilization of the source field in 3-D magnetostatic problems," IEEE Trans. Magn., vol. 34, pp. 2509-2512, Sept. 1998.

[4] A. M. Vishnevsky, A. G. Kalimov, A. A. Lapovok, "Modeling magnetization using Whitney facet elements," IEEE Trans. Magn., vol.38, no.2, pp.489-492, Mar. 2002.

[5] A. M. Vishnevsky, A. J. Lapovok, "Boundary integral computation of electric fields in multielectrode galvanic systems using normally continuous elements," Proc. Inst. Elect. Eng. - Sci. Meas. Technol., vol.141, no.3, pp.145-151, May. 2000.

[6] P. Alotto, F. Desideri, F. Freschi, A. Machio, M. Reptto, "Dual-PEEC modeling of a two-port TEM cell for VHF applications," IEEE Trans. Magn., vol.47, no.5, pp.1486-1489, Fev. 2011.

[7] T-S. Nguyen, J-M. Guichon, O. Chadebec, G. Meunier and B. Vincent, "An independent loops search algorithm for solving inductive PEEC large problems," Progress in Electromagnetic Research M., vol. 23, pp. 53-63, Jan. 2012.

[8] L. Turner, et al., "Workshops and problems benchmarking eddy current codes. TEAM Workshops: Test problem 7," Argonne, Illinois, 1988.

[9] Flux Cedrat. Meylan, France : www.cedrat.com

[10] T-A. Phung, O. Chadebec, G. Meunier, P. Labie, Y. Le Floch, "Automatic cuts for magnetic scalar potential formulation," IEEE Trans. Mag., vol. 41, no. 5, pp. 1668-1671, May. 2005.

[11] T-S. Nguyen, J-M. Guichon, O. Chadebec, P. Labie, and J-L. Coulomb, "Ships magnetic anomaly computation with integral equation and fast multipole method," IEEE Trans. Mag., vol. 47, no. 5, pp. 1414-1417, May. 2011.

[12] R. Albanese, G. Rubinacci, "Integral formulation for 3D eddy current computation using edge elements," Physical Science, Measurement and Instrumentation, Management and Education Reviews, IEE Processing Pt.A, vol.35, no.7, pp.457-462, Sep.1988.

[13] J. Albert, R. Banucu, V. Reinauer, C. Scheiblich, and W. M. Rucker "Comparision of a direct and a vector potential integral equation method for the computation of eddy currents," IEEE Trans. Mag., vol. 48, no. 2, pp. 599-602, Feb. 2012. 